\title{
Diagnóstico de Situación Perinatal
}

\author{
Dres.: Jubiz Hasbún Alfonso; Cardona Toro Luis Eduardo; \\ Restrepo de Ramírez Libia; Manotas Cabarca Rafael de J.; Halpert Ziskind David; \\ Murillo Alvarez Oscar; Callejas José Manuel; Salazar Julio César; \\ Sierra Antonio; Casas Nelly.
}

Publicación Científica presentada al XIV

Congreso Nacional de Obstetricia y Ginecología

\section{Introducción}

Son numerosas las investigaciones epidemiológicas intra e interinstitucionales sobre factores que influyen en el resultado final del proceso reproductivo, que se han publicado en los últimos años. En ellas se ha estudiado con especial interés el período perinatal.

Los datos que tienden a caracterizar la población atendida y evaluar la calidad de la asistencia que a ella se brinda, tienen una importancia particular en la atención médica materno infantil. Caracterizar esta población y evaluar la calidad de la atención médica prestada, era una ambición en nuestro país hace algunos años, hoy, es una necesidad prioritaria y urgente.

La Historia Clínica, como instrumento incuestionable en la práctica de la $\mathrm{Me}$ dicina, puede llegar a ser un medio facilitador del proceso mencionado, tanto en lo que respecta al equipo de atención como a la institución donde ésta se brinda.

El objetivo principal de la aplicación de la Historia Clínica Perinatal, es el de ofrecer al equipo de salud un instrumento que contribuya a elevar la calidad de la asistencia prestada durante el período perinatal.

La Historia Clínica que aquí se presenta, integra los datos materno-fetoneonatales adecuándose a los adelantos científicos producidos en la Obstetricia y en la Neonatología. La implementación de esta Historia en nuestras Instituciones servírá de base para la planificación de la atención de la embarazada $y$ su hijo.

Este diagnóstico de situación perinatal es el resultado de una tarea grupal Ilevada a cabo en el Hospital General de Medellín, Fundación Hospitalaria San Vicente de Paúl y en las Unidades Intermedias de Atención Médica de los Barrios San Cristóbal y Castilla, en estrecha colaboración con todos los miembros de la organización de dichas Instituciones.

Este trabajo demuestra la riqueza de datos que se puede obtener mediante la recolección sistematizada de la información de todos los procesos reproductivos, en una o en varias Instituciones 
que se dediquen a la atención de la madre y su hijo, mediante la utilización del modelo de Historia Clínica Perinatal que se propone.

Estos datos son de valor práctico inmediato para cada una de las 4 Instituciones. Lo anterior, permite tomar decisiones para disminuir el riesgo perinatal y obliga a la revisión y actualización de algunas normas. Además, permite una adecuada utilización de los recursos físicos y materiales, y facilita la capacitación del personal médico y paramédico.

Más allá de la presentación de un diagnóstico de situación perinatal, nuestro objetivo principal es proponer, como un modelo a imitar, la metodología seguida en el presente estudio.

\section{Finalidad del trabajo}

El objetivo de este estudio es determinar algunas características de la población materno-feto-neonatal atendida y de la atención médica brindada en el conjunto de las 4 Instituciones mencionadas.

\section{Fuente de información}

La Historia Clínica Perinatal, recomendada por la O.P.S/O.M.S., fue la fuente principal para obtener los datos que se presentan en esta investigación. Además, hemos tenido como guía en la elaboración de los datos, los dos informes que se han publicado sobre diagnóstico de situación perinatal de la Maternidad Ramón Sardá de Buenos Aires.

\subsection{Características de la Historia Clínica Perinatal}

El modelo normativo utilizado sistematiza la recolección de los datos materno-feto-neonatales más importantes. Su elaboración se hizo tomando en cuenta los objetivos que figuran a continuación:

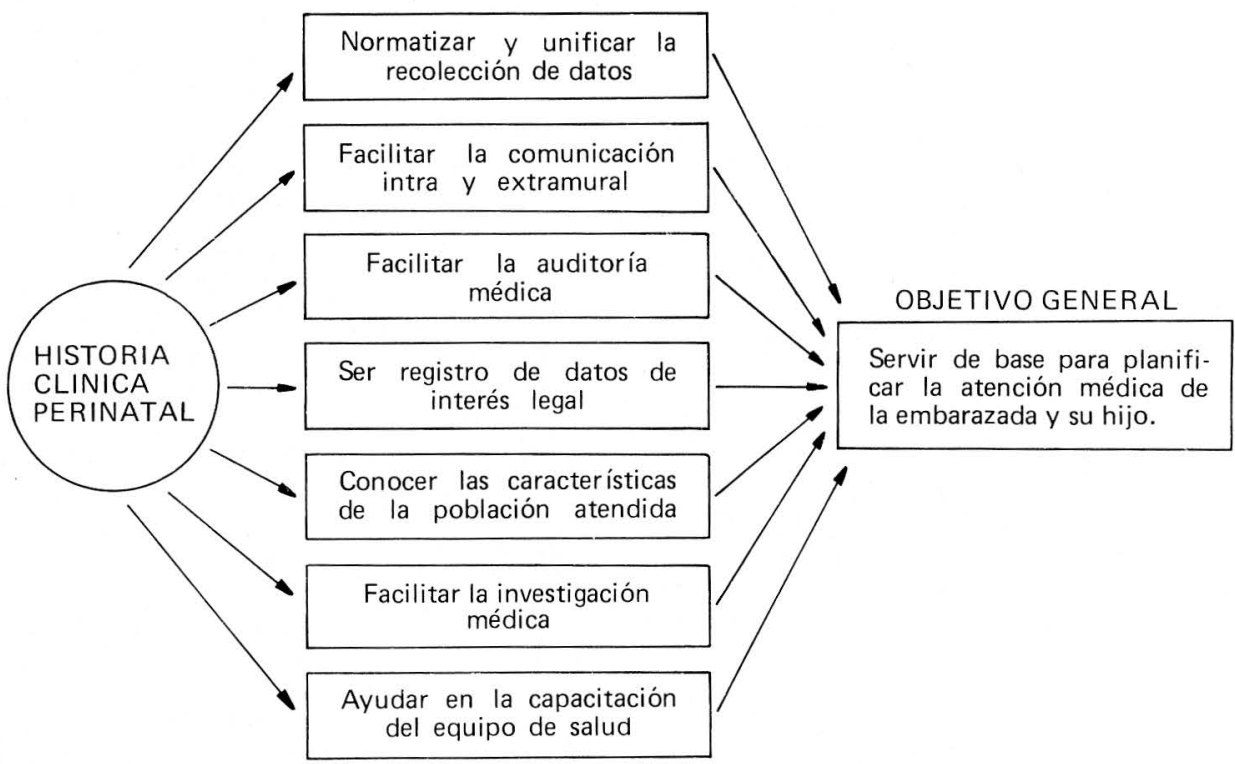


La Historia Clínica Perinatal uniformiza, el contenido de la documentación correspondiente a un acontecimiento obstétrico (embarazo, parto y puerperio) $y$ al recién nacido en el período neonatal inmediato.

Sus formularios contienen un listado de preguntas que, en su mayoría, se contestan con respuestas cerradas. En todos los casos existen, además, espacios libres para los comentarios que se consideren necesarios.

La diagramación general de la historia y el instructivo para su llenado facilita que los datos considerados de importancia sean sistemática y uniformemente recabados y registrados en el momento de la atención.

Esta característica, además de ser de mucho valor como ayuda para el cumplimiento de las normas, constituye un recurso docente en cuanto que estimula su recordación.
El ordenamiento de los datos permite su recolección y volcado en un tiempo más breve y agiliza su ulterior análisis, ya sea por quien los recogió como también por aquel, que no habiéndolo hecho, necesita información incluida en la historia.

La Historia Clínica Perinatal está constituida por cuatro módulos integrados que corresponden a distintas etapas del proceso de atención. Se desarrollan en períodos y lugares distintos y pueden estar a cargo de diferentes miembros del equipo de salud.

La Historia, además, cuenta con un carné perinatal y un resumen estadístico perinatal codificado.

La falta de datos en algún período del proceso de atención médica compromete la calidad de la asistencia de las etapas siguientes.

Esta situación se presenta, por ejemplo, toda vez que el parto no se realiza

\begin{tabular}{|c|c|c|c|}
\hline Modulo & $\begin{array}{l}\text { Etapa del período } \\
\text { perinatal }\end{array}$ & $\begin{array}{c}\text { Lugar de } \\
\text { atención }\end{array}$ & $\begin{array}{c}\text { Responsable de la } \\
\text { atención }\end{array}$ \\
\hline $\begin{array}{l}\text { Prenatal } \\
\text { y puerto } \\
\text { y alto riesgo } \\
\text { (Ambulatorio) }\end{array}$ & $\begin{array}{l}\text { Parto } \\
\text { puerperio }\end{array}$ & $\begin{array}{l}\text { Consulta Prenatal } \\
\text { Bajo y Alto } \\
\text { riesgo }\end{array}$ & $\begin{array}{c}\text { Del control } \\
\text { prenatal }\end{array}$ \\
\hline $\begin{array}{l}\text { Atención } \\
\text { Neonatal }\end{array}$ & $\begin{array}{l}\text { Sala de Partos } \\
\text { y de } \\
\text { puerperio }\end{array}$ & $\begin{array}{c}\text { Del parto y } \\
\text { puerperio }\end{array}$ \\
\hline $\begin{array}{l}\text { Internación } \\
\text { durante } \\
\text { el embarazo }\end{array}$ & $\begin{array}{l}\text { Neonatal } \\
\text { Riesgo } \\
\text { (Internado) }\end{array}$ & $\begin{array}{l}\text { Sala de } \\
\text { - Recepción } \\
\text { Internación } \\
\text { Neonatal } \\
\text { - Alojamiento } \\
\text { Conjunto } \\
\text { Madre - Hijo }\end{array}$ & $\begin{array}{c}\text { Del } \\
\text { Recién } \\
\text { Nacido }\end{array}$ \\
\hline
\end{tabular}




\section{NEXO ENTRE LOS PERIODOS DE ATENCION PRE Y POSTNATAL}

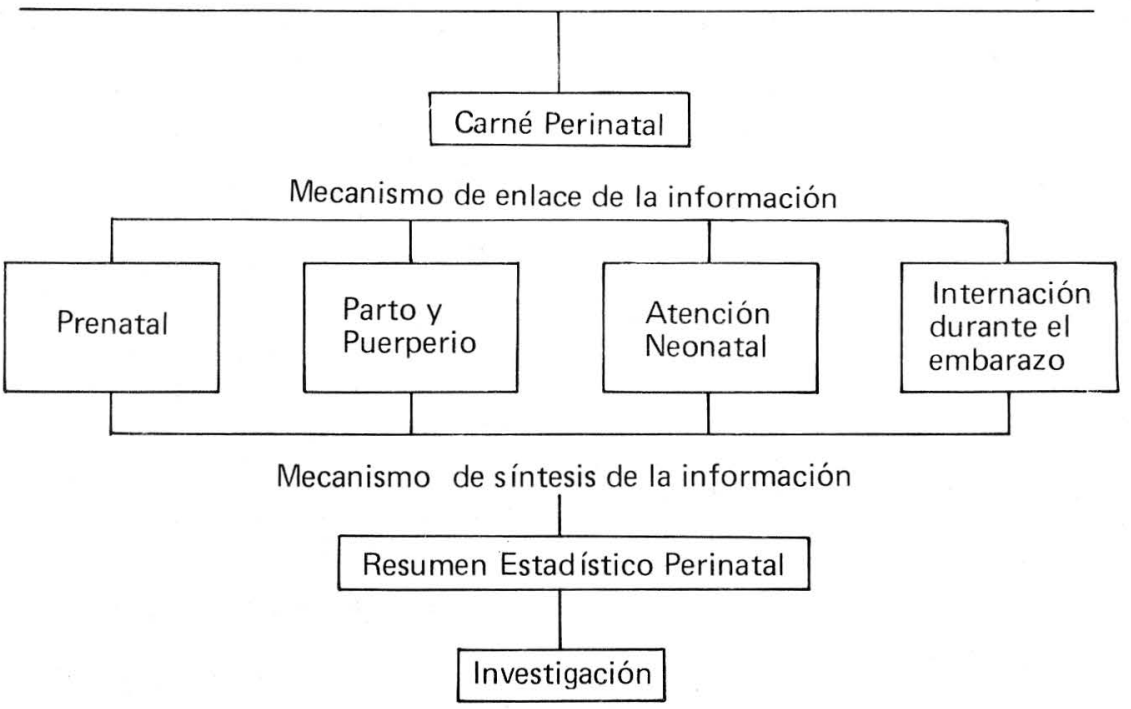

en la misma Institución donde se efectúa el control prenatal. Lo mismo ocurre cuando el control post-parto y pediátrico se realizan fuera de la Institución donde nació el niño.

El Carné Perinatal es un instrumento que constituye un nexo entre los distintos períodos de la atención $y$, por ello, tiende a evitar el problema mencionado.

Este Carné debe estar siempre en poder de la embarazada, quien lo utilizará para toda acción médica que solicite en su estado grávido-puerperal.

\section{Con el Carné Perinatal se asegura:}

3.1.1. Que los datos fundamentales relativos al control prenatal, al ser registrados sistemáticamente en cada consulta, lleguen a manos de quien atiende posteriormente a la embarazada, sea a nivel de otro consultorio externo o en el lugar de internación.
3.1.2. Que los datos de más relevancia de la internación durante el embarazo, parto y postparto lleguen al conocimiento de quien tiene a su cargo el control del puerperio.

3.1.3. Que los datos más importantes para el seguimiento del recién nacido lleguen al conocimiento de quien tiene a su cargo el control del niño.

3.2. Implementación de la Historia Clínica Perinatal en el Hospital General de Medellín, en la Fundación Hospitalaria San Vicente de Paúl y en las Unidades Intermedias de los Barrios Castilla y San Cristóbal.

El 1o. de septiembre de 1980 se implantó la Historia Clínica Perinatal en las 4 Instituciones mencionadas. Para alcanzar el objetivo anterior fue necesario cumplir unas etapas preliminares.

Primero se realizó un seminario en el Departamento de Obstetricia y Gineco- 
logía de la Facultad de Medicina de la Universidad de Antioquia durante los días 27 y 28 de noviembre de 1979. A dicha reunión fueron invitados representantes de las diferentes Instituciones hospitalarias que brindan atención materno-infantil, incluyendo al I.S.S. Después del seminario se impulso la historia en la Fundación Hospitalaria San Vicente de Paúl y durante un mes se realizó una prueba piloto.

En el Hospital General de Medellín tuvo lugar otra reunión preparatoria durante el mes de mayo de 1980. En esta oportunidad, además de los asesores de la O.P.S., se contó con la participación de los representantes del Departamento de Organización y Sistemas de la Universidad de Antioquia.

En julio de 1980 la Junta Directiva de la Asociación Antioqueña de Obstetricia y Ginecología nombra el Comité Coordinador para que se encargara de la organización, preparación y presentación de la ponencia al XIV Congreso Colombiano de Obstetricia y Ginecología. De inmediato el Comité inicia sus funciones y desde la primera reunión plantea las ventajas de un diagnóstico de situación perinatal en 4 Centros Asistenciales de la ciudad de Medellín, utilizando como fuente de información la Historia Clínica Perinatal.

Para la recolección de los datos en los distintos centros, se nombran colaboradores médicos y para-médicos bajo la supervisión del Comité Coordinador.

Durante el mes de agosto de 1980 se realiza una prueba piloto en el Hospital General de Medellín y se ultiman los detalles relacionados con la capacitación del personal.

El 1o. de septiembre de 1980 se inicia la recolección de los datos para el estudio estadístico que hoy presentamos.

\section{Material y Métodos}

En los 4 Centros mencionados se recogió la información entre el 1o. de septiembre de 1980 y el 30 de mayo de 1981.

\subsection{Formulario para la Recolección de los Datos}

Se modificó el Formulario del Resumen Estadístico Perinatal propuesto por los autores de la historia, con las sugerencias técnicas dadas por el Departamento de Organización y Sistemas de la Oficina de Planeación de la Universidad de Antioquia. El formulario de 80 columnas resume los datos más relevantes del proceso reproductivo. La diagramación es clara y sencilla lo que facilita su llenado rápido, utilizando el instructivo que lo acompaña para aclarar eventuales dudas.

La impresión de los nuevos formularios del Resumen Estadístico Perinatal estuvo a cargo de la Editorial de la Universidad de Antioquia.

\subsection{Recolección de los Datos}

El Resumen Estadístico Perinatal se llena antes de que la historia pase al Departamento de Archivos Médicos. Esta labor fue encomendada a enfermeras o secretarias previamente capacitadas en Ios cuatro Centros. La información consignada en estos resúmenes era controlada sistemáticamente por el Comité Coordinador.

Los objetivos de este control fueron:

4.2.1. Verificar la fidelidad de la información recogida.

4.2.2. Asegurar que la totalidad de los partos quedaran registrados.

4.3. Traslado de los Datos a Minidiscos. Control de Consistencia. Adapta- 
ción del Banco de Datos y Procesamiento en los mismos.

Una vez que los resúmenes estadísticos estuvieron completos y debidamente controlados, la información de 13.514 pacientes fue transcrita a minidiscos. (Grabación y verificación).

Posteriormente se hizo la prueba de consistencia de los datos en un computador IBM-370-138 perteneciente al Municipio de Medellín. Los resultados de esta primera crítica fueron revisados por el Comité Coordinador. Luego se corrigió la in. formación grabada en los minidiscos y se realizó una segunda crítica. Una vez depurada la información en los minidiscos, se llevó a una cinta magnética a partir de la cual, se obtuvieron los datos estad ísticos básicos iniciales mediante Ios programas escritos en lenguajes Cobol, Fortran y RPG II, elaborados en el Departamento de Organización y Sistemas de la Universidad de Antioquia.

\section{Resultados}

Los resultados preliminares de este estudio se presentan en gráficas, cuadros y tablas, solamente con las aclaraciones indispensables para su comprensión.

5.1. Datos Preconcepcionales Recabados en la Primera Consulta.

La información que se presenta en el punto 5.1 corresponde a la totalidad del período comprendido entre septiembre 1o. de 1980 y mayo de 1981.
Gráfico No. 1

\section{DISTRIBUCION DE LA EDAD DE LAS EMBARAZADAS MEDELLIN}

Septiembre 1980 - Mayo 1981

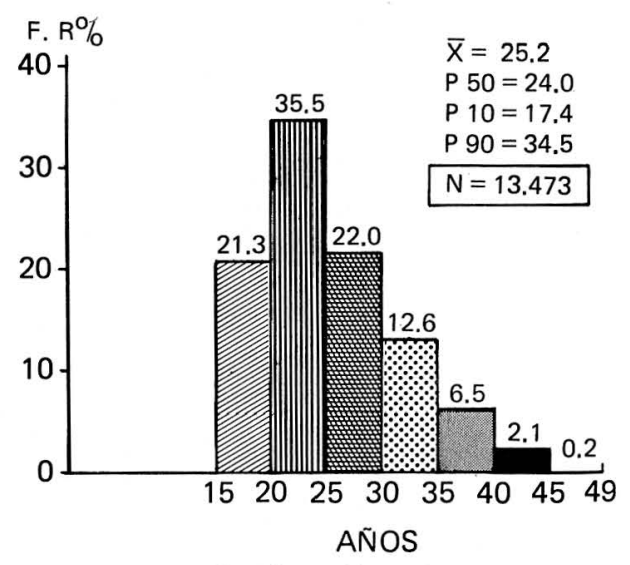

Cuadro No. 1

ESTADO CIVIL DE LAS EMBARAZADAS

\begin{tabular}{|l|r|r|}
\cline { 2 - 3 } \multicolumn{1}{c|}{ Medellín -1981} & N & F.R. $\%$ \\
\hline Casadas & 6.832 & 50.6 \\
\hline Unión Libre & 3.858 & 28.5 \\
\hline Solteras & 2.792 & 20.7 \\
\hline Otros & 28 & 0.2 \\
\hline Total & 13.510 & 100.0 \\
\hline
\end{tabular}

La exclusión ex-profeso de los comentarios que estos datos generan, se ha hecho con la finalidad de dejarlos a cargo del lector. Esta característica de la presentación da, a quien analiza el documento, la libertad suficiente para elaborar conclusiones personales que inciten a la discusión e intercambio de ideas, y ayuden a profundizar el conocimiento de su propia Institución. Quiénes esten interesados en los modelos y esquemas de la Historia Clínica, favor dirigirse a los autores. 
Gŕafico No. 2

\section{INSTRUCCION DE LA EMBARAZADA MEDELLIN}

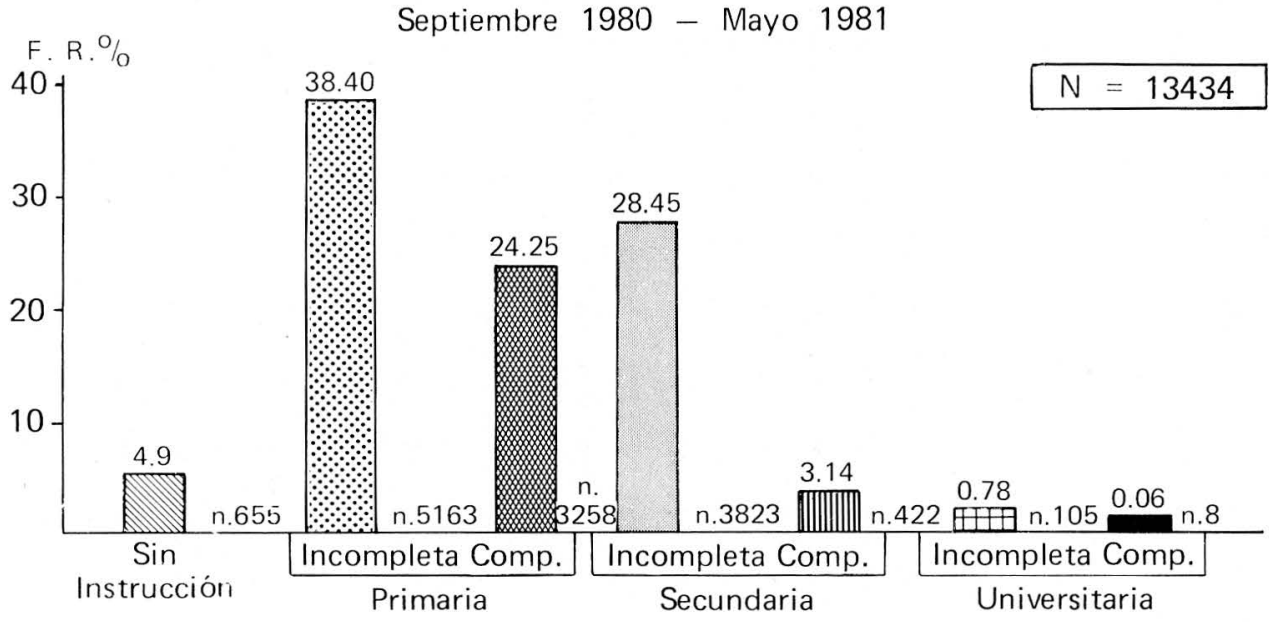

Gráfico No. 3

INSTRUCCION DEL CONYUGE

MEDELLIN

Septiembre 1980 - Mayo 1981

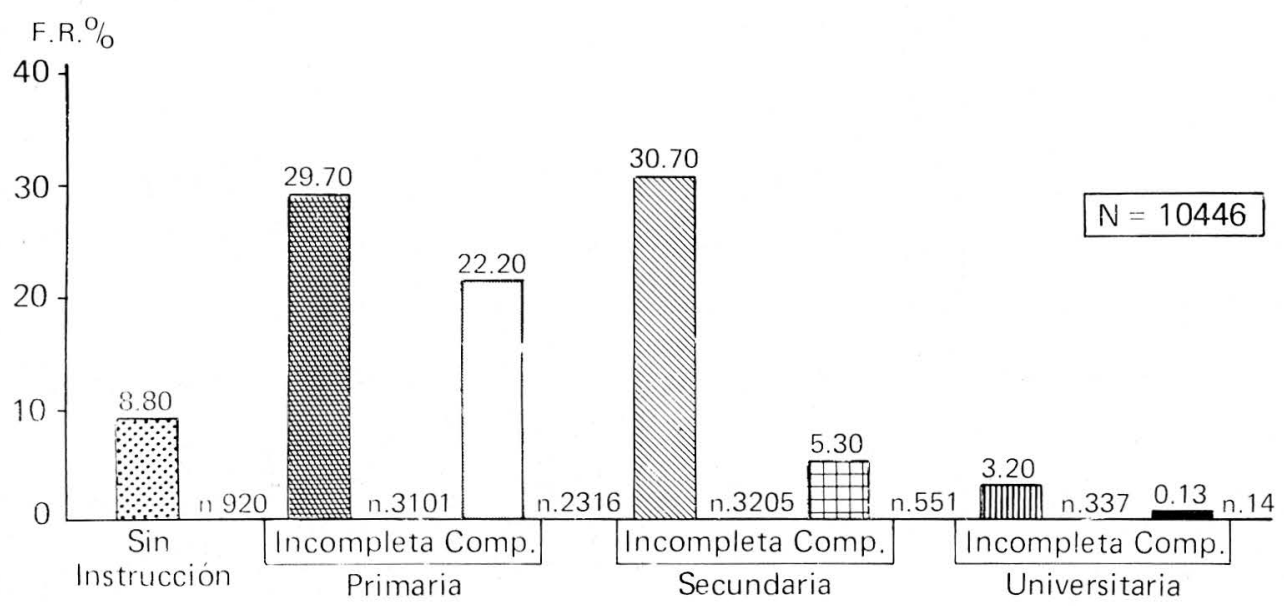


Gráfico No. 4

NIVEL OCUPACIONAL DEL CONYUGE - MEDELLIN
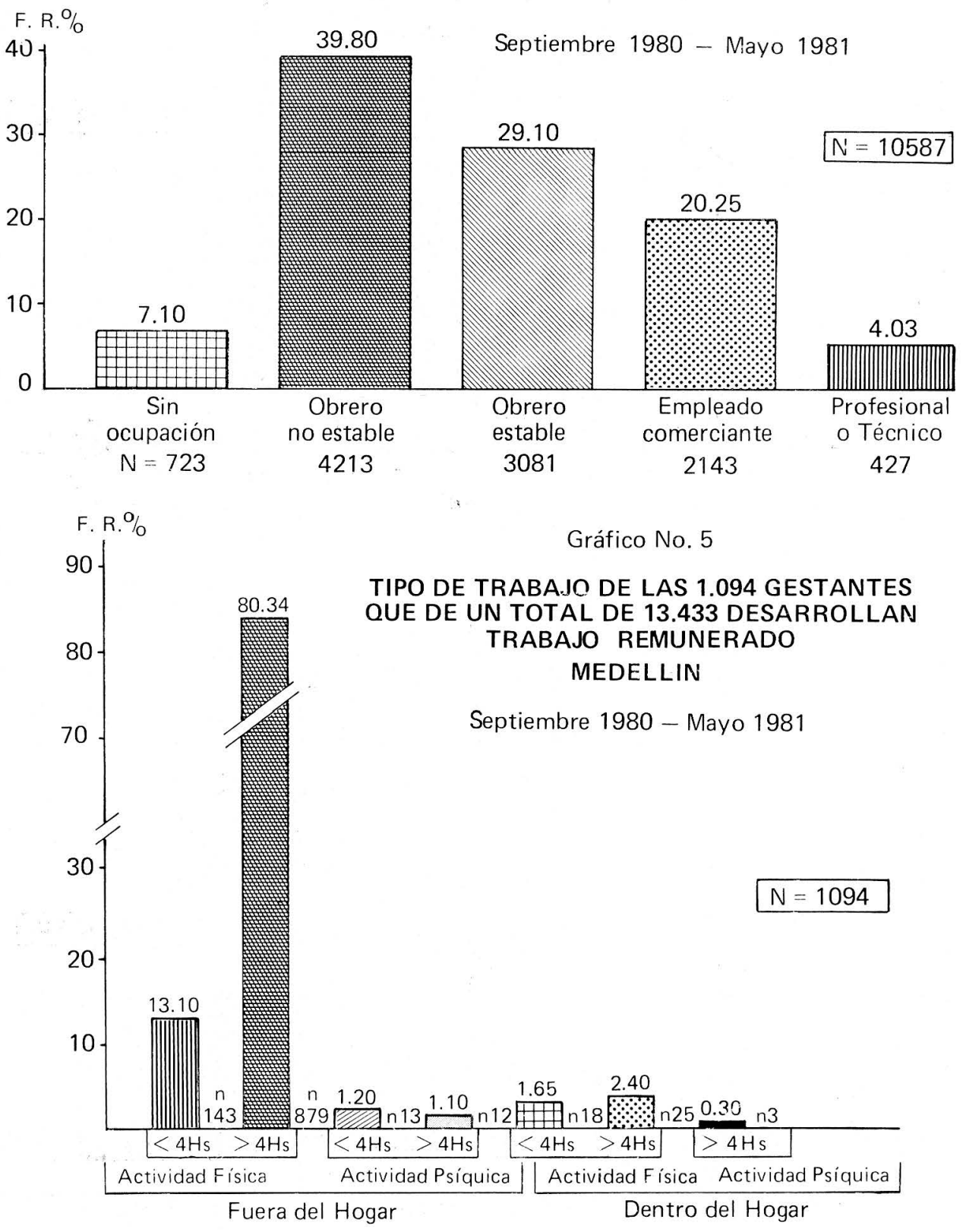


\section{Gráfico No. 6 \\ NUMERO DE PERSONAS QUE HABITAN POR \\ ALCOBA EN LA VIVIENDA DE LA \\ EMBARAZADA \\ MEDELLIN}

Septiembre 1980 - Mayo 1981

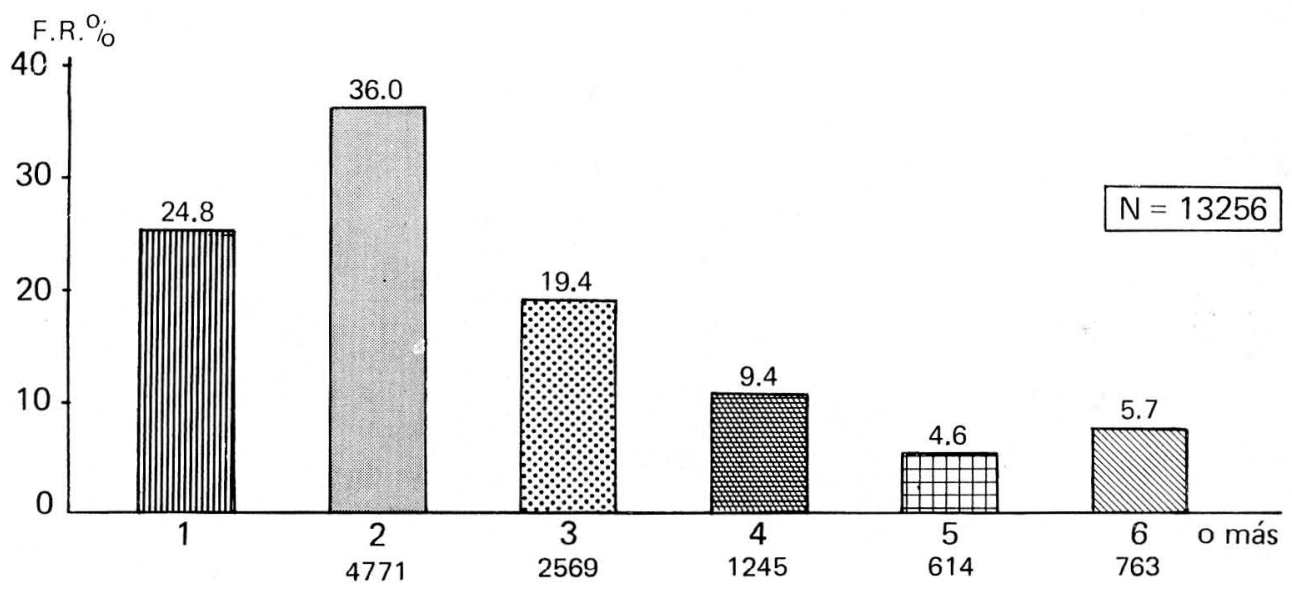

Gráfico No. 7

\section{PESO MATERNO ANTERIOR AL PRESENTE EMBARAZO MEDELLIN}

Septiembre 1980 - Mayo 1981

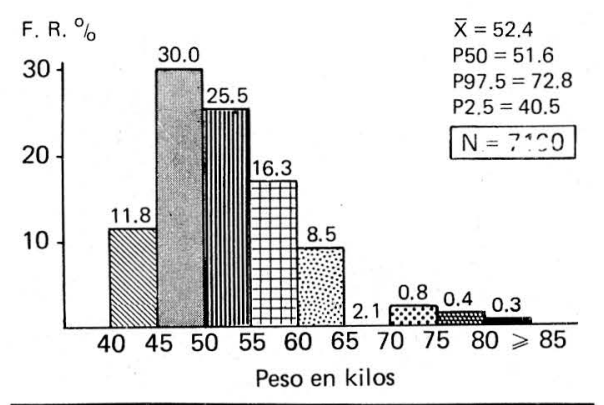

Gráfico No. 8

\section{TALLA DE LAS EMBARAZADAS MEDELLIN}

Septiembre 1980 - Mayo 1981

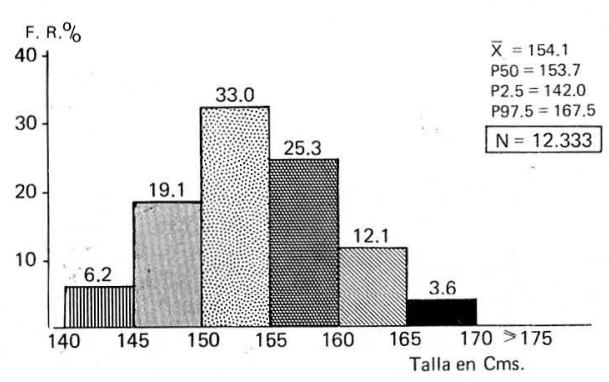


Gráfico No. 9

\section{INTERVALO INTERGENESICO MEDELLIN}

Septiembre 1980 - Mayo 1981

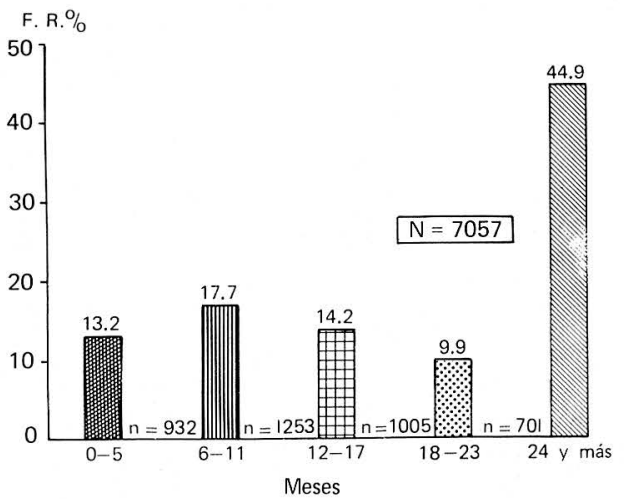

Gráfico No. 10

NUMERO DE

GESTACIONES PREVIAS

MEDELLIN

Septiembre 1980 - Mayo 1981

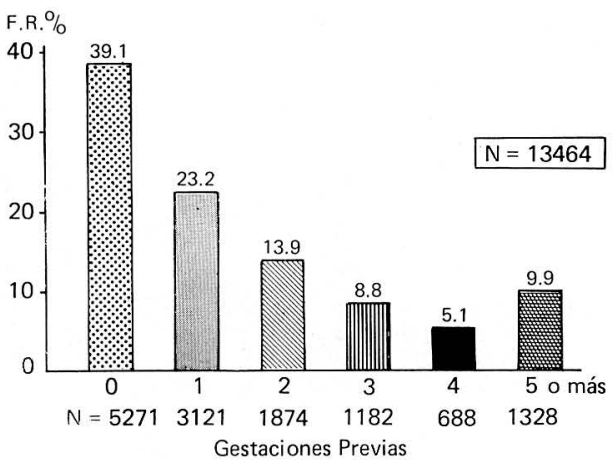

Gráfico No. 11

NUMERO DE CONTROLES PRENATALES

MEDELLIN

Septiembre 1980 - Mayo 1981

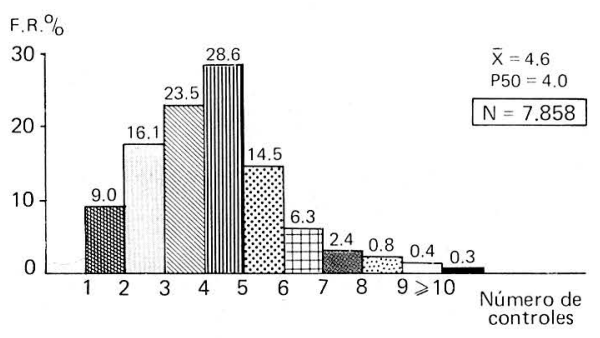

Gráfico No. 12

NUMERO DE CONTROLES

PRENATALES EN GESTANTES

SIN PATOLOGIA DETECTADA MEDELLIN

Septiembre 1980 - Mayo 1981

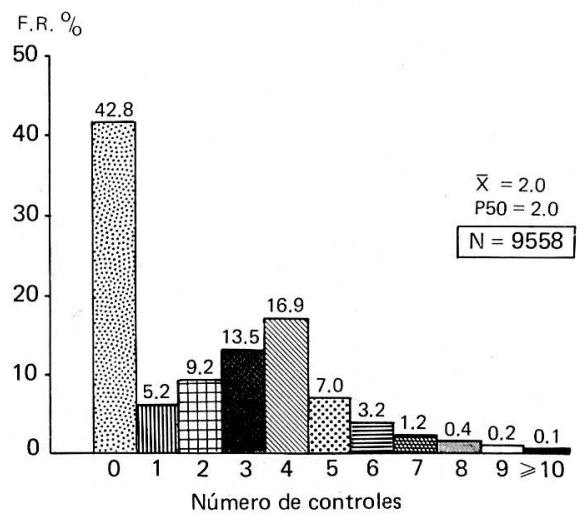


Gráfico No. 12A

\section{NUMERO DE CONTROLES \\ PRENATALES CON \\ PATOLOGIA DETECTADA MEDELLIN}

Septiembre 1980 - Mayo 1981

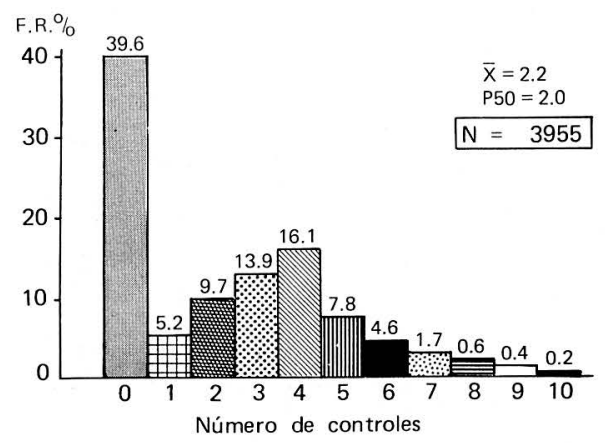

Gráfico No. 13

DURACION TOTAL DE PARTO DESDE 4-5 CMS DE DILATACION MEDELLIN

Septiembre 1980 Mayo 1981

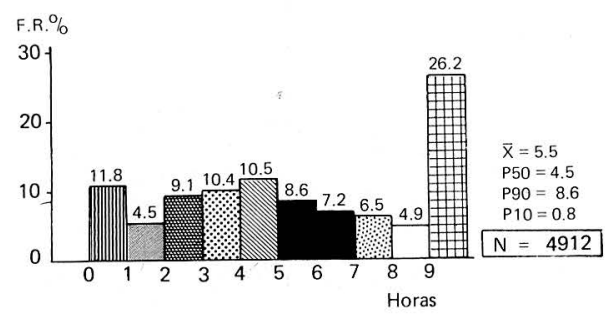

5.2. Control Prenatal

De las embarazadas del estudio, 7.515 $(56 \%)$, tuvieron algún Control Prenatal. El $44 \%$ restantes ( $N$ : 5.900), no tuvieron Control Prenatal.
Número de Controles Prenatales en gestantes con y sin patología detectada. En este último grupo no se incluyen los embarazos gemelares.

En las embarazadas sin patología, la media y la mediana fueron de dos Con. troles Prenatales. En las embarazadas con patología la media fue de 2.2 Controles Prenatales y la mediana de 2.

Cuadro No. 2

EDAD GESTACIONAL A LA PRIMERA CONSULTA MEDELLIN

Septiembre 1980 - Mayo 1981

\begin{tabular}{|c|c|c|c|}
\cline { 2 - 4 } \multicolumn{1}{c|}{} & \multicolumn{3}{c|}{ Trimestre } \\
\cline { 2 - 4 } \multicolumn{1}{c|}{} & 1er. & 2do. & 3er. \\
\hline F.R. $\%$ & 15.5 & 49.0 & 35.5 \\
\hline N & 1.164 & 3.675 & 2.673 \\
\hline
\end{tabular}

Tabla No. 1

DISTRIBUCION DE LA EDAD DE LAS EMBARAZADAS

No. 13.473

\begin{tabular}{|c|r|}
\hline Años & F. A. \\
\hline $15-19$ & 2.738 \\
$20-24$ & 4.784 \\
$25-29$ & 2.959 \\
$30-34$ & 1.704 \\
$35-39$ & 877 \\
$40-44$ & 283 \\
$45-49$ & 28 \\
\hline
\end{tabular}


Tabla No. 2

DISTRIBUCION DE LOS PESOS DE LAS EMBARAZADAS

\begin{tabular}{|r|r|}
\hline Peso .. & F. A. \\
\hline $40-44$ & 847 \\
$45-49$ & 2.147 \\
$50-54$ & 1.826 \\
$55-59$ & 1.169 \\
$60-64$ & 611 \\
$65-69$ & 298 \\
$70-74$ & 151 \\
$75-79$ & 58 \\
$80-84$ & 28 \\
$85-89$ & 25 \\
\hline & 7.160 \\
\hline
\end{tabular}

Tabla No. 3

DISTRIBUCION DE LA TALLA DE LAS EMBARAZADAS

\begin{tabular}{cr} 
Talla & F. A. \\
\hline $140-144$ & 770 \\
$145-149$ & 2.356 \\
$150-154$ & 4.066 \\
$155-159$ & 3.121 \\
$160-164$ & 1.494 \\
$165-169$ & 442 \\
$170-174$ & 57 \\
$>175$ & 27 \\
& 12.333
\end{tabular}

Tabla No. 4

PESO DE LOS RECIEN NACIDOS

\begin{tabular}{crr} 
Peso al Nacer & \multicolumn{1}{c}{$N$} & F.R. $\%$ \\
\hline $500-999$ gramos & 49 & 0.4 \\
$1.000-1.499$ gramos & 81 & 0.6 \\
$1.500-1.999$ gramos & 205 & 1.5 \\
$2.000-. .499$ gramos & 902 & 7.0 \\
$2.500-2.999$ gramos & 3.735 & 28.0 \\
$3.000-3.499$ gramos & 5.492 & 41.2 \\
$3.500-3.999$ gramos & 2.397 & 18.0 \\
$4.000-4.499$ gramos & 398 & 3.0 \\
$4.500-4.999$ gramos & 71 & 0.5 \\
\hline Total & 13.330 & 100.0 \\
\hline
\end{tabular}

Gráfico No. 14

EDAD GESTACIONAL AL PARTO POR AMENORREA MEDELLIN

Septiembre 1980 - Mayo 1981

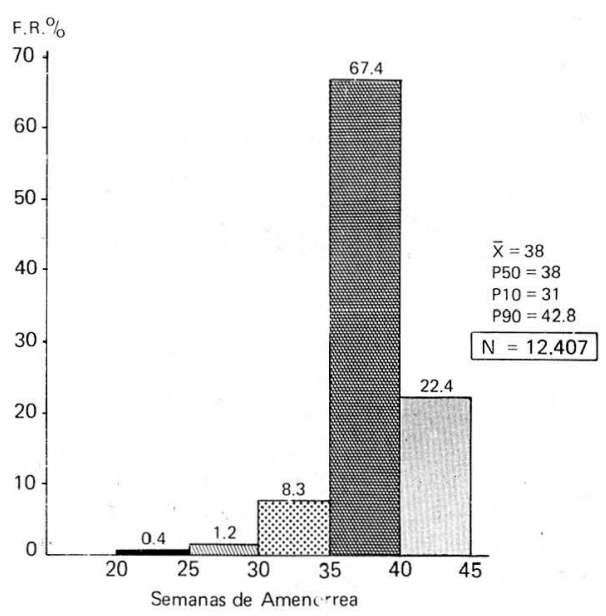


Gráfico No. 15

\section{PESO AL NACER EN EMBARAZOS UNICOS}

MEDELLIN

F.R.\% Septiembre 1980 - Mayo 1981

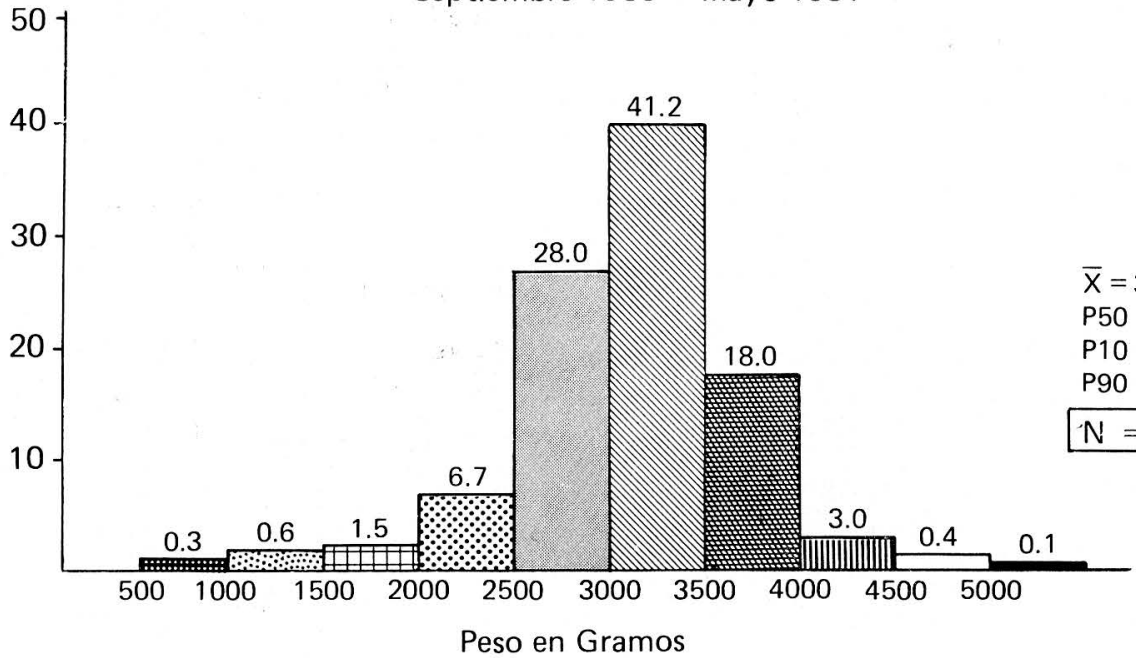

Gráfico No. 16

MORTALIDAD FETAL INTERMEDIA + TARDIA

$Y$ NUMERO DE CONTROLES PRENATALES

Septiembre 1980 - Mayo 1981

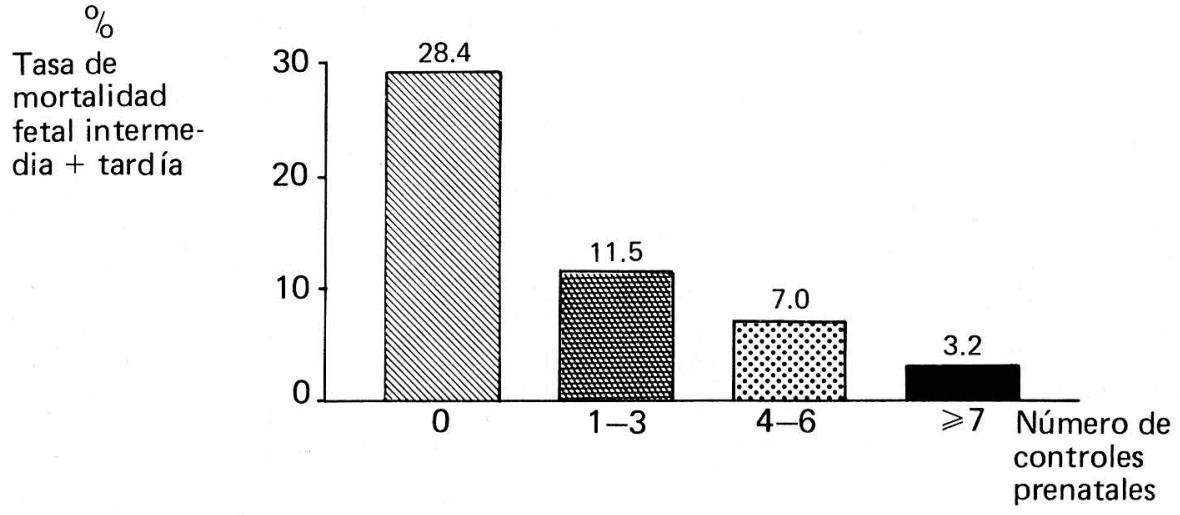


Cuadro No. 3

HABITO DE FUMAR

EN LAS EMBARAZADAS

MEDELLIN

Septiembre 1980 - Mayo 1981

\begin{tabular}{|c|c|c|c|}
\hline & \multirow[b]{2}{*}{$\mathrm{N}$} & \multirow[b]{2}{*}{ F.R. $\%$} \\
\hline & & & \\
\hline & No fumadoras & 8.647 & 68.0 \\
\hline \multirow{5}{*}{$\begin{array}{l}\mathrm{F} \\
\mathrm{U} \\
\mathrm{M} \\
\mathrm{A} \\
\mathrm{D} \\
\mathrm{O} \\
\mathrm{R} \\
\mathrm{A} \\
\mathrm{S}\end{array}$} & $1-5$ cigarrillos/día & 2.312 & 18.2 \\
\hline & $6-10$ cigarrillos/día & 942 & 7.4 \\
\hline & $11-15$ cigarrillos/día & 98 & 0.8 \\
\hline & $16-20$ cigarrillos/día & 708 & 5.6 \\
\hline & Total & 12.707 & 100.0 \\
\hline
\end{tabular}

Cuadro No. 5

ADMINISTRACION

DE OXITOCINA

DURANTE EL PARTO

(SE EXCLUYEN LOS

FETOS MUERTOS)

MEDELLIN

Septiembre 1980 - Mayo 1981

\begin{tabular}{|l|r|c|}
\cline { 2 - 3 } \multicolumn{1}{c|}{} & N & F.R. $\%$ \\
\hline Sin oxitocina & 12.409 & 93.4 \\
\hline Oxitocina Dilatante & 871 & 6.6 \\
\hline Oxitocina P. Expulsivo & 12 & 1.0 \\
\hline $\begin{array}{c}\text { Oxitocina Dilatante }+ \\
\text { Expulsivo }\end{array}$ & 0 & 0 \\
\hline \multicolumn{1}{|c|}{ Total } & 13.292 & 100.0 \\
\cline { 2 - 3 }
\end{tabular}

Cuadro No. 4

FORMA DEL COMIENZO

DEL PARTO EXCLUYENDO

LOS FETOS MUERTOS

MEDELLIN

Septiembre 1980 - Mayo 1981

\begin{tabular}{|l|r|r|}
\cline { 2 - 3 } \multicolumn{1}{c|}{} & \multicolumn{1}{c|}{$\mathrm{N}$} & F.R. $\%$ \\
\hline Espontáneo & 12.464 & 97.7 \\
\hline Inducido & 294 & 2.3 \\
\hline Total & 12.758 & 100.0 \\
\hline
\end{tabular}

Cuadro No. 6

ANALGESICOS

$Y$ TRANQUILIZANTES

ADMINISTRADOS DURANTE

EL PARTO (SE EXCLUYEN

LOS FETOS MUERTOS)

MEDELLIN

Septiembre 1980 - Mayo 1981

\begin{tabular}{|c|c|c|}
\hline & $\mathrm{N}$ & F.R. $\%$ \\
\hline Ninguna & 12.739 & 96.8 \\
\hline Meperidina & 358 & 2.7 \\
\hline Diazepan & 40 & 0.3 \\
\hline Diazepan - otras & 17 & 0.13 \\
\hline Mep. + Diazepan & 9 & 0.07 \\
\hline Total & 13.163 & 100.0 \\
\hline
\end{tabular}


Cuadro No. 7

ANESTESIA DURANTE EL PARTO (SE EXCLUYEN LOS

FETOS MUERTOS)

MEDELLIN

Septiembre 1980 - Mayo 1981

\begin{tabular}{|l|r|r|}
\cline { 2 - 3 } \multicolumn{1}{c|}{} & $\mathrm{N}$ & F.R. $\%$ \\
\hline Sin anestesia & 5.187 & 39.0 \\
\hline Perineal & 5.086 & 38.3 \\
\hline General & 1.143 & 8.6 \\
\hline Raquídea & 888 & 6.7 \\
\hline Peridural & 754 & 5.7 \\
\hline Otras & 167 & 1.2 \\
\hline Pudenda & 58 & 0.5 \\
\hline Total & 13.283 & 100.0 \\
\hline
\end{tabular}

Cuadro No. 9

FORMA DE TERMINACION DEL PARTO

(SE EXCLUYEN

FETOS MUERTOS)

MEDELLIN

Septiembre 1980 - Mayo 1981

\begin{tabular}{|l|r|c|}
\cline { 2 - 3 } \multicolumn{1}{c|}{} & \multicolumn{1}{c|}{$N^{\prime}$} & F.R. \% \\
\hline Espontáneo & 10.333 & 77.3 \\
\hline $\begin{array}{l}\text { Cesárea electiva }+ \\
\text { Intraparto }\end{array}$ & 1.803 & 13.5 \\
\hline Fórceps bajo & 986 & 7.4 \\
\hline Fórceps medio & 165 & 1.2 \\
\hline Asistencia pelviana & 66 & 0.5 \\
\hline Extracción pelviana & 13 & 0.1 \\
\hline Total & 13.366 & 100.0 \\
\hline
\end{tabular}

Cuadro No. 8

TIEMPO TRANSCURRIDO ENTRE LA ROTURA DE

MEMBRANAS Y EL NACIMIENTO

MEDELLIN

Septiembre 1980 - Mayo 1981

\begin{tabular}{|r|r|r|}
\cline { 2 - 3 } \multicolumn{1}{c|}{} & \multicolumn{1}{c|}{$N$} & F.R. $\%$ \\
\hline Menos de 5 horas & 10.785 & 84.0 \\
\hline Entre 5 y 23 horas & 1.621 & 12.6 \\
\hline 24 y más horas & 440 & 3.4 \\
\hline Total & 12.846 & 100.0 \\
\hline
\end{tabular}

Cuadro No. 10

PUNTAJE DE APGAR

AL PRIMER MINUTO

MEDELLIN

Septiembre 1980 - Mayo 1981

\begin{tabular}{|c|c|c|}
\hline Puntaje & N & F.R. ${ }^{\circ} \%$ \\
\hline $1-3$ & 322 & 2.5 \\
\hline $4-6$ & 697 & 5.2 \\
\hline 7 y más & 12.275 & 92.3 \\
\hline Total & 13.294 & 100.0 \\
\hline
\end{tabular}


Cuadro No. 11

PUNTAJE DE APGAR

AL QUINTO MINUTO

MEDELLIN

Septiembre 1980 - Mayo 1981

\begin{tabular}{|c|r|c|}
\hline Puntaje & N & F.R. $\%$ \\
\hline $1-3$ & 63 & 0.5 \\
\hline $4-6$ & 166 & 1.2 \\
\hline 7 y más & 13.068 & 98.3 \\
\hline Total & 13.297 & 100.0 \\
\hline
\end{tabular}

Cuadro No. 14

INCIDENCIA DE PATOLOGIA PUERPERAL

MEDELLIN

Septiembre 1980 - Mayo 1981

\begin{tabular}{|l|r|c|}
\cline { 2 - 3 } \multicolumn{1}{c|}{} & $\mathrm{N}$ & F. R. \% \\
\hline Normales & 12.991 & 96.2 \\
\hline Infección & 334 & 2.5 \\
\hline $\begin{array}{l}\text { Hemorragia antes de } \\
\text { dos horas }\end{array}$ & 161 & 1.2 \\
\hline $\begin{array}{l}\text { Hemorragia después } \\
\text { de dos horas }\end{array}$ & 9 & 0.1 \\
\hline \multicolumn{1}{|c|}{ Total } & 13.991 & 100.0 \\
\hline
\end{tabular}

Cuadro No. 12

\section{APGAR SEGUN TERMINACION DEL PARTO AL PRIMER MINUTO MEDELLIN}

Septiembre 1980 - Mayo 1981

\begin{tabular}{|c|c|c|c|c|c|c|}
\cline { 2 - 7 } \multicolumn{1}{c|}{} & Espontáneo & Fórceps bajo & Fórceps medio & Cesárea & $\begin{array}{c}\text { Asistencia } \\
\text { pelviana }\end{array}$ & $\begin{array}{l}\text { Extracción } \\
\text { pelviana }\end{array}$ \\
\hline Puntaje & F. R. $\%$ & F. R. $\%$ & F. R. $\%$ & F. R. $\%$ & F. R. $\%$ & F. R. $\%$ \\
\hline $1-3$ & 1.1 & 4.0 & 6.1 & 7.7 & 7.8 & 23.1 \\
\hline $4-6$ & 2.7 & 15.5 & 29.2 & 10.6 & 21.9 & 30.8 \\
\hline 7 y más & 96.2 & 80.5 & 64.7 & 81.7 & 70.3 & 46.1 \\
\hline Total & 100.0 & 100.0 & 100.0 & 100.0 & 100.0 & 100.0 \\
\hline
\end{tabular}

\section{Discusión}

La Historia Clínica Perinatal que se presenta, integra las distintas etapas del proceso reproductivo. El Carné Perinatal sirve como mecanismo de enlace de la información, y facilita al personal médico y paramédico, el conocimiento de los datos relevantes de la embarazada, cuando su control prenatal, se realiza en lugar diferente al sitio donde se atiende el parto $y$ el recién nacido.

El Resumen Estadístico Perinatal es de gran importancia porque sintetiza la información recabada en la Historia 
Cuadro No. 13

\section{APGAR SEGUN TERMINACION DEL PARTO AL OUINTO MINUTO MEDELLIN}

Septiembre 1980 - Mayo 1981

\begin{tabular}{|l|c|c|c|c|c|c|}
\cline { 2 - 7 } \multicolumn{1}{c|}{} & Espontáneo & Fórceps bajo & Fórceps medio & Cesárea & $\begin{array}{c}\text { Asistencia } \\
\text { pelviana }\end{array}$ & $\begin{array}{c}\text { Extracción } \\
\text { pelviana }\end{array}$ \\
\hline Puntaje & F. R. $\%$ & F. R. $\%$ & F. R. $\%$ & F. R. $\%$ & F. R. $\%$ & F. R. $\%$ \\
\hline $1-3$ & 0.3 & 0.2 & 1.2 & 1.1 & 3.1 & 7.7 \\
$4-6$ & 0.7 & 1.3 & 1.8 & 3.8 & 3.1 & 7.7 \\
7 y más & 99.0 & 99.5 & 97.0 & 95.1 & 93.8 & 84.6 \\
\hline Total & 100.0 & 100.0 & 100.0 & 100.0 & 100.0 & 100.0 \\
\hline
\end{tabular}

Clínica y facilita, de esta manera, la recolección de los datos para el análisis estad ístico, la adecuación de normas de asistencia y la investigación en el área perinatal.

Queremos resaltar la importancia del trabajo armónico en grupo, como factor importante para impulsar la implementación de la Historia y recoger información valiosísima en diferentes sitios de atención Materno Infantil.

La metodología que aquí presentamos es fácilmente reproducible en cualquiera de los distintos niveles de atención médica de Colombia.
Finalmente, queremos insistir en la necesidad de caracterizar la Población Materno Infantil de Colombia para planificar $y$ adecuar su atención.

\section{Conclusiones}

7.1. La Historia Clínica Perinatal, permite al personal que hace administración en los programas Materno Infantiles, adecuar la prestación de servicios oportuna y real a la embarazada y su hijo.

7.2. Los datos que se presentan, constituyen una primera aproximación de un diagnóstico de situación Perinatal.

\section{DIAGNOSIS OF A PERINATAL SITUATION}

\section{Conclusions}

The clinical perinatal record allows the administrative personnel in the mother and Infant programs to optimize the services rendered to the pregnant mother and her child.
The data presented constitutes a first approximation of a diagnosis of the perinatal situation. 


\section{Bibliografía}

1. BELIZAN, J.M. et al. Historia Clínica Perinatal. Propuesta de un Modelo Ministerio de Bienestar Social: Secretaría de Estado de Salud Pública. Dirección Nacional de Maternidad e Infancia. Argentina. OPS. OMS. Publicado por el CLAP. Nov. 1976.
2. DIAZ, A.G. et al. Diagnóstico de Situación Perinatal. Resultados Obtenidos con la Aplicación de la Historia Clínica Perinatal en el Hospital Sardá de Buenos Aires. Datos correspondientes a 9.758 partos consecutivos desde julio 1976 a diciembre de 1978. Publicación Científica 846 CLAP. OPS. OMS. Nov. 1979. 